\title{
Benefical Effects of Sigma-1 Agonist Fluvoxamine for Tardive Dyskinesia and Tardive Akathisia in Patients with Schizophrenia: Report of Three Cases
}

\author{
Yakup Albayrak $^{1 \otimes}$ and Kenji Hashimoto ${ }^{2}$ \\ ${ }^{1}$ Department of Psychiatry, Medical Faculty, Namık Kemal University, Tekirdag, Turkey \\ ${ }^{2}$ Division of Clinical Neuroscience, Chiba University Center for Forensic Mental Health, Chiba, Japan
}

Fluvoxamine is a selective serotonin reuptake inhibitor that is approved for psychiatric disorders such as major depressive episodes and obsessive-compulsive disorder. Beside inhibition of serotonin reuptake, fluvoxamine is also a potent agonist of endoplasmic reticulum (ER) protein sigma-1 receptors, which play a role in the pathophysiology of a number of psychiatric and neurodegenerative disorders. This report presents beneficial effects of sigma-1 agonist fluvoxamine on hyperkinetic movement disorders such as tardive dyskinesia and tardive akathisia. Fluvoxamine might be a novel treatmet approach in the treatment of hyperkinetic movement disorders.

Psychiatry Investig 2013;10:417-420

Key Words Sigma receptor, Fluvoxamine, Akathisia, Dyskinesia.

\section{INTRODUCTION}

Fluvoxamine is a selective serotonin reuptake inhibitor (SSRI) that is approved for psychiatric disorders such as major depressive episodes by the European Medicines Agency and obsessive-compulsive disorder by the US Food and Drug Administration. Beside inhibition of serotonin reuptake, fluvoxamine is also a potent agonist of endoplasmic reticulum (ER) protein sigma-1 receptors, which play a role in the pathophysiology of a number of psychiatric and neurodegenerative disorders. ${ }^{1}$ Here, we report three schizophrenic patients who benefited from sigma-1 agonist fluvoxamine in terms of tardive dyskinesia (TD) and tardive akathisia (TA).

\section{CASE}

\section{Case 1}

Ms B, a 43-year-old woman, had been diagnosed with schi-

Received: December 11, 2012 Revised: February 5, 2013

Accepted: March 25, 2013 Available online: October 24, 2013

$\triangle$ Correspondence: Yakup Albayrak, MD

Department of Psychiatry, Medical Faculty, Namık Kemal University, Namık Kemal Mahallesi, Kampus Caddesi, Tekirdag, Turkey

Tel: +905056355434, Fax: +02822509928, E-mail: dr.fuge@hotmail.com

(a) This is an Open Access article distributed under the terms of the Creative Commons Attribution Non-Commercial License (http://creativecommons.org/licenses/by$\mathrm{nc} / 3.0$ ) which permits unrestricted non-commercial use, distribution, and reproduction in any medium, provided the original work is properly cited. zophrenia for 22 years. She had been treated with conventional antipsychotics as haloperidol, zuclopenthixol, chlorpromazine for almost 12 years. For the last 10 years, she had been treated with various atypical antipsychotics such as olanzapine, aripiprazole, ziprasidone. She has be given quetiapine $800 \mathrm{mg} /$ day and biperiden $4 \mathrm{mg} /$ day for last two years. She had been also diagnosed as tardive dyskinesia for almost 10 years and her treatment was switched to atypical antipsychotics from typical antipsychotics for last 10 years. The results of brain magnetic resonance imaging (MRI), encephalography (EEG), blood chemistry, a complete blood count, and thyroid function tests were all normal. On psychiatric and physical examination, fatigue, difficulty concentrating and choreic movements on fingers of bilateral upper limbs were noted. Ms A was diagnosed with schizophrenia, residual type and medication induced movement disorder (tardive dyskinesia) according to the Diagnostic and Statistical Manual of Mental Disorders, Fourth Edition, Text Revision (DSM-IV-TR). ${ }^{2}$ Ms A scored 6 on the Abnormal Involuntary Movement Scale (AIMS). ${ }^{3}$ Fluvoxamine $100 \mathrm{mg} /$ day was added to her treatment. At the fourth week, the dyskinesia had improved gradually based on scores of 2 on the AIMS.

\section{Case 2}

Mr M, a 41-year-old man, had been diagnosed with schizo- 
phrenia for 18 years. He had been treated with conventional antipsychotics as haloperidol, pimozide and zuclopenthixol for almost 13 years. For the last 5 years, he has been treated with olanzapine $20 \mathrm{mg} /$ day and biperidene $4 \mathrm{mg}$ /day. The results of MRI, EEG, blood chemistry, a complete blood count, and thyroid function tests were all normal. On psychiatric and physical examination, avolution, mood, anhedonia, insomnia, fatigue, difficulty concentrating and choreic movements on bilateral upper limbs were noted. The choreic movements has been existent for nearly seven years. Mr M was diagnosed with schizophrenia, residual type and medication induced movement disorder (tardive dyskinesia) according to the DSM-IV-TR. ${ }^{2}$ Ms A scored 11 on the AIMS. ${ }^{3}$ Fluvoxamine $100 \mathrm{mg} /$ day was added to treatment. At the fourth week, the dyskinesia had improved gradually and observed to be improved in terms of tardive dyskinesia with on score of 3 on the AIMS.

\section{Case 3}

Ms M, a 36-year-old woman, had been diagnosed with schizophrenia for 6 years. She had been treated with quetiapine $600 \mathrm{mg} /$ day for 5 years. For the last 1 year, she has been treated with olanzapine $20 \mathrm{mg} /$ day. She began complaining of severe motor restlessness and an unbearable state of never being able to sit still for one month before her last visit for regular outpatient clinic control. The results of brain MRI, EEG, blood chemistry, a complete blood count, and thyroid function tests were all normal. On psychiatric and physical examination, anxiety, agitation and akathisia were noted. Ms $\mathrm{M}$ was diagnosed with schizophrenia, residual type; and medication induced movement disorder (tardive akathisia) according to the DSMIV-TR. ${ }^{2}$ On the Barnes Akathisia Rating Scale (BARS), ${ }^{4}$ global score was 5 (severe akathisia). Fluvoxamine $100 \mathrm{mg} /$ day was added to her treatment. Three weeks after adding fluvoxamine $100 \mathrm{mg} /$ day treatment, there was no reemergence of akathisia or other extrapyramidal side effects.

\section{DISCUSSION}

TD is characterized by involuntary, repetitive, purposeless movements that can affect different parts of the body., ${ }^{5,6} \mathrm{It}$ is a well-recognized complication of conventional antipsychotics and usually occurs after several years of treatment. Sometimes antidepressants or calcium channel blockers may be responsible. ${ }^{7}$ Classically, the tongue, face, and neck muscles are involved, but the extremity muscles and the muscles controlling body posture and breathing can also be affected. ${ }^{8}$ The pathophysiology of tardive dyskinesia is complex and remains unclear. ${ }^{9}$ The most common theory on the pathophysiology of tardive dyskinesia supposes a supersensitivity of dopamine
(DA) receptors from prolonged receptor blockade or upregulation of these receptors. ${ }^{10}$ Other theories include striatal disinhibition of the thalamocortical pathway from an imbalance of the D1 and D2 receptors caused by the chronic use of DA blocking agents. Additionally, neurodegeneration secondary to lipid peroxidation or excitotoxic mechanisms may be responsible. ${ }^{11}$ Because of the relatively poor understanding of the pathophysiology, no effective treatment for TD is currently available. The best approach to management remains prevention, including restricting the use of antipsychotic medications to established indications and alternative treatments when possible. When neuroleptic medications are needed, they should be prescribed in the smallest therapeutic doses for the shortest possible time. ${ }^{12}$ There is no definitive treatment for tardive dyskinesia, although tetrabenazine ameliorates tardive dyskinesia most effectively. Vitamin B6, vitamin E, donepezil, levetiracetam, and botulinum toxin are other treatment options. $^{12}$

Akathisia literally means "inability to remain seated". Most investigators agree that there are two aspects of akathisia: a subjective report of restlessness and inner tension and the objective manifestations of restlessness in the form of semipurposeful or purposeless movements of the limbs. ${ }^{13}$ Akathisia associated with neuroleptics can be subtyped according to its onset and its duration. Most research on akathisia deals with acute subtype and detailed information on other subtypes is limited. If akathisia occurs three months after exposuring the neuroleptic, it can be considered as TA. The exact pathophysiology of TA is unknown and speculative. Dopaminergic mechanisms have recieved much attention. Tardive or withdrawal onset, supression with neuroleptics and response to dopamine depleting drugs such as tetrabenazine all suggest that the DA receptor supersensitivty hypothesis which has been proposed for TD. ${ }^{14}$

Fluvoxamine is shown to be a potent sigma-1 receptor agonist. ${ }^{1}$ A positron emission tomography study using a selective sigma-1 receptor agonist, SA4503, showed that fluvoxamine bound to sigma-1 receptors in the living human brain at therapeutic doses, suggesting that sigma-1 receptors play a role in the mechanism of action of fluvoxamine. ${ }^{15}$ There is also conflicting evidence that sigma-1 receptor agonists increase and reduce dopamine levels in the striatum. ${ }^{16}$ The sigma-1 receptors are concentrated in specific areas of the limbic system and brainstem motor structures of the human brain. The density of sigma-1 receptors is moderate to high in the caudate, putamen, septum, nucleus accumbens, and amygdala in rats and humans. ${ }^{16}$ Currently, there are some case reports showing that fluvoxamine was effective in the hyperkinetic movement disorders. Furuse and Hashimoto reported that fluvoxamine was effective in the blonanserin-induced ${ }^{17}$ and aripiprazole-in- 
duced ${ }^{18}$ akathisia in patients with schizophrenia. Fluvoxamine was reported to be effective for treating both hemiballism and depression in a patient with depressive disorder, and it was suggested that fluvoxamine might act as reducer of dopamine in striatal area. ${ }^{19}$ Fluvoxamine was also found to be beneficial for reducing choreic movements in a patient with Huntington's Disease. ${ }^{20}$ We reported that fluvoxamine was effective in the treatment of duloxetine induced TD. ${ }^{21}$ Recently, we reported five patients with postpsychotic depression of schizophrenia who benefited from fluvoxamine in terms of TD..$^{22}$ Precise mechanisms underlying the effects of fluvoxamine for TD and TA are currently unclear, but the chaperone activity of sigma-1 receptors on ER might play a role in its mechanism. ${ }^{1,23,24}$ Sigma-1 receptor agonists, including fluvoxamine, might modulate dopaminergic neurotransmission in the limbic area via chaperone activity because sigma-1 receptors are known to modulate a number of neurotransmitter systems. ${ }^{25}$

Accumulating evidence suggests the role of ER stress in the pathophysiology of neurodegenerative disorders such as TD. ${ }^{26,27}$ The major function of molecular chaperones at the ER is to lead proper folding of newly synthesized proteins. These actions of molecular chaperones for ER stress are important for cells to prevent accumulation of toxic protein aggregates, thus facilitating cellular survival under cellular stress. ${ }^{28}$ The mechanism regulating chaperone activity of the sigma-1 receptors involves the physical protein-protein interaction between sigma-1 receptors and another ER chaperone, immunoglobulin heavy chain-binding protein BiP/GRP78. ${ }^{23}$ When the sigma-1 receptors form a conjugate with BiP/GRP78 , the chaperone activity is minimized. In contrast, the sigma-1 receptors dissociated from BiP/ GRP78 exert the maximum chaperone activity. Importantly, several synthetic compounds (e.g., fluvoxamine) possessing the agonist property of the sigma- 1 receptors promote the dissociation of the sigma- 1 receptors from BiP; thus sigma-1 receptor agonists gain the chaperone activity of the sigma-1 receptors. ${ }^{24,25}$ It is known that haloperidol is a potent antagonist at sigma-1 receptor on ER. As mentioned above, sigma-1 receptor agonists, such as fluvoxamine, promote the dissociation of the sigma-1 receptor from its binding parter BiP, thus maximizing the chaperone activity of the sigma-1 receptor. In contrast, sigma-1 receptor antagonists, such as haloperidol, reinforce the association, thus blocking the action of sigma-1 receptor agonists. ${ }^{24,29}$ Therefore, it is possible that long-term blockage of sigma-1 receptor by typical antipsychotics such as haloperidol may contribute to the ER stress which may be involved in the incidence of motor side effects. Furthermore, it is likely that chaperone activity of sigma-1 receptor by fluvoxamine may improve the motor side effects by the regulation for ER stress. Considering the neurodegenerative and exitotoxic theories of tardive dyskinesia as well as tardive akathisia, the sigma-1 receptor agonist fluvoxamine might produce therapeutic effects via chaperone activity on ER.

This report presents beneficial effects of sigma- 1 agonist fluvoxamine on hyperkinetic movement disorders such as $\mathrm{TD}$ and TA. Fluvoxamine might be a novel treatmet approach in the treatment of hyperkinetic movement disorders. Nonetheless, further detailed, double-blind studies should clarify the potential use of fluvoxamine in the treatment of hyperkinetic movement disorders.

\section{REFERENCES}

1. Hashimoto K. Sigma-1 receptors and selective serotonin reuptake inhibitors: clinical implications of their relationship. Cent Nerv Syst Agents Med Chem 2009;9:197-204.

2. American Psychiatric Assosiation. Diagnostic and Statistical Manual of Mental Disorders, 4 th Edition, text revsion. Washington DC: American Psychiatric Assosiation; 2000.

3. Lavalaye J, Sarlet A, Booij J, Linszen DH, Reneman L, Gersons BP, et al. Dopamine transporter density in patients with tardive dyskinesia: A single photon emission computed tomography study. Psychopharmacology (Berl) 2001;155:107-109.

4. Barnes TR. A rating scale for drug-induced akathisia. Br J Psychiatry 1989;154:672-676.

5. Jeste DV, Rockwell E, Harris MJ, Lohr JB, Lacro J. Conventional vs. newer antipsychotics in elderly patients. Am J Geriatr Psychiatry 1999; 7:70-76.

6. Laporta M, Archambault D, Ross-Chouinard A, Chouinard G. Articulatory impairment associated with tardive dyskinesia. J Nerv Ment Dis 1990;178:660-662.

7. Jankovic J. Tardive syndromes and other drug-induced movement disorders. Clin Neuropharmacol 1995;18:197-214.

8. Schwartz M, Silver H, Tal I, Sharf B. Tardive dyskinesia in northern Israel: preliminary study. Eur Neurol 1993;33:264-266.

9. Casey DE. Tardive dyskinesia: pathophysiology and animal models. J Clin Psychiatry 2000;61(Suppl 4):5-9.

10. Lavalaye J, Sarlet A, Booij J, Linszen DH, Reneman L, Gersons BP, et al. Dopamine transporter density in patients with tardive dyskinesia: A single photon emission computed tomography study. Psychopharmacology (Berl) 2001;155:107-109.

11. Brasic JR, Bronson B. Tardive dyskinesia. Available at: emedicine. medscape.com/article/1151826-overview. Accessed May 24, 2010.

12. Soares KV, McGrath JJ. The treatment of tardive dyskinesia--a systematic review and meta-analysis. Schizophr Res 1999;39:1-16.

13. Sachdev PS. Neuroleptic-induced movement disorders: an overview. Psychiatr Clin North Am 2005;28:255-274.

14. Sachdev PS. The classification of akathisia. Mov Disord 1995;10:235237.

15. Ishikawa $M$, Ishikawa $M$, Ishii $K$, Kimura $Y$, Sakata $M$, Naganawa $M$, et al. High occupancy of sigma-1 receptors in the human brain after single oral administration of fluvoxamine: a positron emission tomography study using [11C]SA4503. Biol Psychiatry 2007;62:878-883.

16. Gudelsky GA. Effects of sigma receptor ligands on the extracellular concentration of dopamine in the striatum and prefrontal cortex of the rat. Eur J Pharmacol 1995;24:223-228.

17. Furuse T, Hashimoto K. Fluvoxamine for blonanserin-associated akathisia in patients with schizophrenia: report of five cases. Ann Gen Psychiatry 2010;9:17.

18. Furuse T, Hashimoto K. Fluvoxamine for aripiprazole-associated akathisia in patients with schizophrenia: a potential role of sigma-1 receptors. Ann Gen Psychiatry 2010;9:11. 
19. Cayköylü A., Albayrak Y, Uğurlu GK, Ekinci O. Beneficial effects of fluvoxamine for hemiballism in a patient with depressive disorder: a case report. Acta Neurol Belg 2011;111:62-65.

20. Albayrak Y, Ugurlu GK, Ugurlu M, Cayköylü A. Beneficial effects of fluvoxamine for chorea in a patient with huntintong's disease: a case report. Prim Care Companion CNS Disord 2012;14.

21. Albayrak Y, Ekinci O. Duloxetine associated tardive dyskinesia resolved by fluvoxamine: case report. J Clin Psychopharmacol 2012;32:723-724

22. Albayrak Y, Hashimoto K. Beneficial effects of the sigma-1 agonist fluvoxamine for tardive dyskinesia in patients with postpsychotic depressive disorder of schizophrenia: report of 5 cases. Primary Care Companion CNS Disord 2012;14.

23. Schroder M, Kaufman RJ. The mammalian unfolded protein response. Annu Rev Biochem. 2005;74:739-789.

24. Hayashi T, Tsai SY, Mori T, Fujimoto M, Su TP. Targeting ligand-operated chaperonesigma-1 receptors in the treatment of neuropsychiatric disorders. Expert Opin Ther Targets 2011;15:557-577.

25. Hayashi T, Su TP. An update on the development of drugs for neuropsychiatric disorders: focusing on the sigma 1 receptor ligand. Expert Opin Ther Targets. 2008;12:45-58.

26. Hashimoto K, Ishiwata K. Sigma receptor ligands: possible application as therapeutic drugs and as radiopharmaceuticals. Curr Pharm Des 2006;12:3857-3876

27. Lindholm D, Wootz H, Korhonen L. ER stress and neurodegenerative diseases. Cell Death Differ 2006;13:385-392.

28. Doyle KM, Kennedy D, Gorman AM, Gupta S, Healy SJ, Samali A. Unfolded proteins andendoplasmic reticulum stress in neurodegenerative disorders. J Cell Mol Med 2011;15:2025-2039.

29. Hashimoto K. Sigma-1 receptor chaperone and brain-derived neurotrophic factor: Emerging links between cardiovascular disease and depression. Prog Neurobiol 2013;100:15-29. 